East African Medical Journal Vol. 81 No. 2 February 2004

LEISHMANIA MAJOR-PHLEBOTOMUS DUBOSCQI INTERACTIONS: INHIBITION OF ANTI-LPG ANTIBODIES AND CHARACTERISATION OF TWO PROTEINS WITH SHARED EPITOPES

W.K. Tonui, BSc, MSc, PhD, CBiol MIBiol, Research Officer; P.M. Ngumbi, BSC, MSC., Senior Research Officer; S.S. Mpoke, BSC, MSC., Principal Research Officer, Centre for Biotechnology Research and Development, Kenya Medical Research Institute, Nairobi, Kenya; A.S. Orago, BSC, MSC, Professor of Immunology, Kenyatta University, Nairobi, Kenya; P.A. Mbati, BSC, MSC, PhD., Professor of Immunology, University of the North, Phuthaditjhaba, South Africa; S.J. Turco, Professor of Biochemistry, Kentucky Medical Centre, Lexington, Kentucky, USA and G.M. BSC, MSC, PhD., Mkoji, Chief Research Officer and Director, Centre for Biotechnology Research and Development, Kenya Medical Research Institute, Nairobi, Kenya

Request for reprints to: Dr. W.K. Tonui, Centre for Biotechnology Research and Development, Kenya Medical Research Institute, P.O. Box 54840, Nairobi, Kenya

\title{
LEISHMANIA MAJOR-PHLEBOTOMUS DUBOSCQI INTERACTIONS: INHIBITION OF ANTI-LPG ANTIBODIES AND CHARACTERISATION OF TWO PROTEINS WITH SHARED EPITOPES
}

\author{
W.K. TONUI, P.M. NGUMBI, S.S. MPOKE, A.S. ORAGO, P.A. MBATI, \\ S.J. TURCO and G.M. MKOJI
}

\begin{abstract}
Objectives: To assess the effect of monoclonal antibodies (MABS) raised against $L$. major derived LPG on $L$. major development in vitro and in its natural vector $P$. duboscqi. Also determine whether LPG molecule and the sand fly the gut Iysates have shared epitopes.

Design: A laboratory based study.

Setting: Colony bred $P$. duboscqi sand flies and all other experiments were done under laboratory conditions.

Methods: Laboratory reared sand flies were allowed to feed beneath a blood filled membrane feeder containing $1 \times 10^{6}$ amastigotes in $20 \mu \mathrm{l}$ mixed with $0.5 \mathrm{ml}$ of defibrinated rabbit blood with a 1:100 dilution of anti-LPG MABS. Control blood contained a similar number of amastigotes but no MABS. At least five female previously fed sand flies were later dissected on days two, four, and six post-feeding and examined for promastigote forms and parasite loads in the sand fly mid gut. In vitro, the same number of amastigotes in $100 \mu$ l complete Schneider's Drosophila medium was mixed in a 96 well plate with either 100 $\mu$ l of 1: 100 anti-LPG MABS, 1:1000 anti LPG MABS or undiluted sera from L. major infected mice. The control well contained a similar number of amastigotes but no antibodies added. Following an overnight incubation in a $\mathrm{CO}_{2}$ incubator at $37^{\circ} \mathrm{C}$ and growth at $26^{\circ} \mathrm{C}$, parasites were assessed at 3, 6 and 24 hour intervals for changes in their developmental forms.

Results: 1:100 dilution of anti-LPG MABS when mixed with amastigotes were effective in reducing $L$. major development at the early log phase or procyclic stage both in vitro and within the sand fly $(\mathbf{p}<0.05)$. The control cultures or sand flies that fed on amastigotes alone and no MABS supported full parasite development up to the metacyclic stage. Results also showed that flies, which had fed on MABS, showed low parasitemia levels of $2+$, compared to a high density of $4+$ for their controls $(p<0.5)$.

Conclusions: These findings showed that anti-LPG MABS were effective in reducing sand fly infections. This study also showed that $P$. duboscqi gut lysates and proteins present in $L$. major-derived LPG share two common proteins of molecular weights $105 \mathrm{kDa}$ and $106 \mathrm{kDa}$. Further analysis of these individual proteins from the gut should be studied with a view of determining their vaccine potential.
\end{abstract}

\section{INTRODUCTION}

Leishmaniasis is a vector-borne disease in which Leishmania parasites are transmitted by the bite of phlebotomine sand flies. In the vertebrate host, Leishmania parasites survive and multiply intracellularly in mononuclear phagocytes as a flagellated amastigotes, about 2 to $54 \mu \mathrm{m}$ in diameter. These cells include macrophages and dendritic cells, and more recently the fibroblasts, which were also shown to be able to harbour parasites(1). The intracellular amastigote form can be maintained in vitro at $37^{\circ} \mathrm{C}$ but transforms to a flagellated promastigote form within the sand fly or at $26^{\circ} \mathrm{C}$ in culture(2). Female sand flies are haematophagous and require a blood meal every 4-5 days and if fed on infected hosts may transmit the disease to new hosts on subsequent feeding. The promastigote parasite form progresses through a series of morphologically distinct developmental stages in the sand fly until it reaches the infectious metacyclic 
stage(2). The first developmental forms are the procyclics, which are short ellipsoid promastigotes of about 6-8 $\mu \mathrm{m}$ body lengths. These procyclics later transform into nectomonads, haptomonads and finally the metacyclics(2).

The cell surface of Leishmania promastigote stage is coated by a protective glycocalyx that comprises a number of glycosylphosphatidylinositol (GPI)-anchored glycoproteins, a complex of free GPI-anchored lipophosphoglycan (LPG), and a family of free GPI's (termed glycolinositophospholipids (GIPLs)(3). The GPIanchored glycoproteins include an abundant metalloproteinase, termed gp63 or leishmaniolysin, and the promastigote surface antigen (PSA2/gp46) family of glycoproteins(4). The complete structure of these molecules has now been established for several species(3). The LPGs and GIPLs function as protective molecules and act as developmentally regulated virulence factors involved in the recognition and attachment of host macrophages as well as binding the gut of the sand fly vector(5). LPG forms a dense glycocalyx that covers the entire cell surface and contains the carbohydrate motif (specific for the particular species of Leishmania) necessary for attachment to the sand fly mid gut(6). Leishmania strains and mutants deficient in LPG biosynthesis are unable to successfully infect sand flies $(6,7)$.

Research in our laboratory has previously shown that polyclonal sera from animals immunised with Leishmania-derived antigens affect vector functions in several ways: lyses of gut epithelium, reduction in parasite loads and transmission; and an increase in sand fly fecundity and mortality rates. Furthermore, the LPG molecule was found to be an excellent candidate as a transmission blocking vaccine against $L$. major infections(8).

Monoclonal antibodies (MABS) raised against L. donovani have also been shown to be more effective at inhibiting L. major development in P. duboscqi than those raised against $L$. major, $L$. tropica or $L$. aethiopica(9). This study sought to investigate the effect of MABS raised against Leishmania majorderived LPG on the development of L. major in vitro and in its natural vector P. duboscqi Neveu Le maire (Diptera: Psychodidae). Determination of whether the Leishmania parasite has molecules of similar molecular weight with gut lysates from its vector was also pursued.

\section{MATERIALS AND METHODS}

Production of monoclonal antibodies against Leishmania major-derived lipophosphoglycan (LPG): This was done as previously described(10). Briefly, BALB/c mice were injected intraperitoneally with $12.5 \mu \mathrm{g}$ of purified $L$. major-derived LPG, in $0.2 \mathrm{ml}$ sterile phosphate buffered saline (PBS), pH 7.4. After three weeks, the mice were injected similarly with $25 \mu \mathrm{g}$ LPG and finally boosted with the same dose intravenously after another three weeks. Three days after the final immunisation, the spleen was removed, a cell suspension made and hybridomas produced using standard fusion protocols(11) with SP2/0 parentalmyeloma cells(12) as the fusion partner. Anti-LPG producing hybridomas were selected by indirect enzyme-linked immunosorbent assay (ELISA) using pure LPG as solid-phase antigen. These MAB-producing hybridomas were inoculated into BALB/c mice and potent IgM antibodies harvested from ascites of these mice. These specific $L$. major-LPG monoclonal antibody (coded WIC 79.3) was then diluted to the desired concentration before use.

Preparation of L. major infected blood: This was done as previously described(9). Briefly, BALB/c mice infected subcutaneously with L. major were sacrificed and their swollen footpads removed. Footpad biopsies were sterilized with $70 \%$ ethanol, left to dry and then excised under sterile conditions. The biopsies were trimmed and transferred into a Tenbroeck tissue grinder containing phosphate buffered saline (PBS) mixed with $25 \mu \mathrm{l} / \mathrm{ml}$ penicillin, $250 \mu \mathrm{g} / \mathrm{ml}$ streptomycin and $500 \mu \mathrm{g} / \mathrm{ml} 5-$ fluorocytosine arabinoside. Biopsies were grounded completely and left to stand in an ice bucket for 15 minutes. The supernatant homogenate were centrifugally washed thrice at 3000 revolutions per minute (rpm) for 10 minutes at $4^{\circ} \mathrm{C}$. Amastigotes that sedimented were re-suspended in $100 \mu \mathrm{l}$ PBS and then counted using a haemocytometer. Viability of amastigotes was confirmed using the in vitro transformation method. A million $\left(1 \times 10^{6}\right)$ amastigotes in $20 \mu \mathrm{l}$ were mixed with $0.5 \mathrm{ml}$ of defibrinated rabbit blood containing a 1:100 or 1:1000 dilution of anti-LPG MABS or undiluted sera from L. major infected mice. The mixture was then vortex mixed for sand fly feeding experiments. Control blood contained a similar number of amastigotes but no MABS added.

Effects of anti-LPG MABS on the development of $L$. major in vitro: Amastigotes in $100 \mu \mathrm{l}$ complete Drosophila medium were mixed in a 96 well plate with either $100 \mu 1$ of 1: 100 anti LPG MABS, 1:1000 anti LPG MABS, undiluted sera from mice previously infected with L. major (polyclonal sera) and controls. The control well contained a similar number of amastigotes but no serum from infected animals or anti-LPG MABS added. These were incubated in a $\mathrm{CO}_{2}$ incubator overnight at $37^{\circ} \mathrm{C}$. The following day parasites were transferred to an incubator and allowed to continue growing at $26^{\circ} \mathrm{C}$. Aliquots from each experimental well were later done at 12, 24 and 36-hour intervals and their smears made on a microscope slide. These parasites were later fixed in methanol and stained using Giemsa's stain and observed under a light microscope for changes in their developmental forms.

Sand flies and Infection: A colony of Phlebotomus duboscqi Neveu Le Maire (Diptera: Psychodidae) that originated from sand flies collected in animal burrows and from termite hill ventilation shafts in Rabai area near the town of Marigat in Baringo district, Rift Valley province in Kenya, was raised at the Kenya Medical Research Institute's (KEMRI) insectaries. The procedure followed to establish and maintain a breeding colony of P. duboscqi from these field-captured females has previously been described(13).

Infection of sand flies was done as previously described (9). Briefly, the membrane through which P. duboscqi fed through were prepared from shaved skins of Swiss Albino mice. These were used to cover glass feeders into which either blood containing amastigotes and MABS or control infected blood were added. Five minutes before sand flies were allowed to feed, the mouse skin covered glass membrane 
feeders were attached to a circulating water bath and left to warm up to $37^{\circ} \mathrm{C}$. Two groups of $120,2-5$ day old unfed female P. duboscqi were aspirated and placed in $30 \mathrm{ml}$ plastic feeding cups filled with fabric-screen lids ( 12 holes per linear $\mathrm{cm})$. The outer space of the screen lids of feeding cups were pressed beneath the blood-filled membrane feeder with one x106 amastigotes with MABS and the other beneath the membrane feeder containing blood mixed with amastigotes only (control). Sand flies were left to feed ad libitum for one hour uninterrupted at room temperature. After feeding, sand flies were transferred into an insectary at $25 \pm 1^{\circ} \mathrm{C}$ and $\mathrm{RH}$ $90 \%$ for 24 hours. Engorged sand flies were given a drop of sterile sugar syrup as a carbohydrate supplement until they were dissected.

Assessment of parasite development within the sand fly: In order to determine the presence or absence of promastigotes, at least five engorged female sand flies were dissected on days two, four, and six post-feeding and examined for promastigote forms as previously described(2). Individual female flies were dissected in a drop of $0.15 \mathrm{M}$ sodium chloride $(\mathrm{NaCl})$, and their guts examined in wet preparations for parasites. Where parasites were seen, their locations within the guts were noted. In order to ascertain parasite forms and to enumerate their numbers in the sand flies, slides used in dissections were air-dried, fixed in absolute methanol and stained in Giemsa's stain. The slides were examined under the light microscope for the presence or absence of nectomonads, haptomonads, paramastigotes and metacyclic promastigotes(2). Total parasitemia were an addition of all parasite forms counted, and the infectivity rates established using published grading systems(14).

Preparation of Leishmania major and sand fly gut antigens: Leishmania major (Strain IDUB/KE/83= NLB-144) serially maintained in BALB/c mice were grown to stationary phase in NNN/Schneider's Drosophila medium. On harvesting, parasites were centrifuged at 2,500 rpm and the pellet washed three times in PBS. To identify the concentration that was to give $30 \mu \mathrm{g}$ when solubilised, parasites reconstituted in $5 \mathrm{ml}$ PBS were Iysed by freeze thawing to release all the proteins. After four cycles of freeze thawing, the parasites were span at $30,000 \mathrm{~g}$ for 30 minutes and the supernatant used as the soluble antigen. Sand fly guts dissected from unfed 3-5 dayold $P$. duboscqi were homogenised in cold, sterile PBS and their protein content determined using the Biorad protein assay. Protein estimation using this assay was done as per the manufacturer's instructions. From these experiments it was determined that $1 \times 10^{6} / \mathrm{ml}$ parasites and 70 sand fly guts were sufficient to yield $30 \mu \mathrm{g}$ of proteins when solubilised. Thus 80 guts and $1 \times 10^{6}$ L. major parasites in $30 \mu l$ of sample buffer respectively, were used in loading the gels during sodium dodecyl sulphate-polyacrylamide gel electrophoresis (SDS-PAGE).

Sample preparation and gel electrophoresis: Whole promastigote lysate were prepared by solubilising in vitro stationary-phase L. major promastigotes (NLB-144) in sample buffer, containing $2 \%$ sodium dodecyl sulphate (SDS), $5 \%$ B-mercaptoethanol and $10 \%$ glycerol or NuPAGE sample buffer(15). The samples were run using the NuPAGE Tris glycine electrophoresis system according to the manufacturer's manual (Invitrogen Life Technologies Ltd, USA). Briefly, before loading, the samples were first prepared by adding $0.5 \mathrm{M}$ dithiothreitol (DTT) reducing agent to a sample constituted to the desired $0.03 \mathrm{mg} / \mathrm{ml}$ in NuPAGE sample buffer and ultra pure water. The sample was vortex mixed and heated for 10 minutes at $70^{\circ} \mathrm{C}$. After heating the samples were vortex mixed again and loaded onto a NuPAGE gel in a Novex Mini-Cell electrophoretic system containing NuPAGE running buffer for 50 minutes. The gel was removed and silver stained using SilverXpress staining kit under the manufacturer's instructions (Novex).

Western blotting: Separated proteins were immunoblotted onto nitrocellulose membranes using highconcentration glycerin buffer in NuPAGE mini trans-blot transfer system(16). Strips of nitrocellulose membrane were incubated with a 1: 1000 dilution of anti-L. major derived LPG (WIC 79.3) for 1 hour (15). After three 5 min washes in $0.1 \%$ Tween 20 and $150 \mathrm{mM} \mathrm{NaCI}$ in $100 \mathrm{mM}$ Tris-HCL, $\mathrm{pH} 7.5$ (TTBS), the strips were incubated with 1:1000 dilution of peroxidase conjugated rabbit anti-mouse $\mathrm{IgG}$ for one hour before washing as before. The strips were washed further in distilled water and colour development was achieved by reaction with BCIP/NBT phosphatase substrate (Kirkegaard and Perry Laboratories, USA). The reaction was thereafter stopped after 10 minutes with cold distilled water.

Statistical Analysis: Chi-square $\left(\mathrm{X}^{2}\right)$ comparison tests were used to compare sand fly infectivity rates and analysis of variance (ANOVA) used to compare the experimental and the control groups.

\section{RESULTS}

Effects of anti-LPG MABS on L. major development in vitro: A summary of results following incubation of $L$. major amastigotes with 1:100 anti-LPG MABS, 1:1000 anti-LPG MABS, undiluted mouse (polyclonal) sera or in culture medium in vitro is given in Table 1). Briefly, the results showed that after incubation at $37^{\circ} \mathrm{C}$ all the experimental wells had mainly the amastigote form (100\%) of the parasite in culture. After 12 hours of incubation at $26^{\circ} \mathrm{C}$, parasites previously mixed with 1:100 anti-LPG MABS showed only the shorter early procyclic promastigotes $(100 \%)$ in culture, while those previously mixed with undiluted mouse sera, 1:1000 anti-LPG MABS showed a mixture of early $\log$ phase procyclics $(40 \%)$ and the longer, broader procyclic promastigotes $(50 \%)$ and few nectomonads $(10 \%)$. At this stage the control wells showed the longer, broader procyclic promastigotes $(15 \%)$ but mainly nectomonads (80\%) and fewer haptomonads (5\%). At 24 hours in culture, the control wells showed very few of the longer, broader procyclic promastigotes $(5 \%)$ and nectomonads $(20 \%)$ but the majority of parasites were haptomonads $(75 \%)$. Parasites previously mixed with undiluted mouse sera also showed the longer, broader procyclics (10\%), nectomonads $(20 \%)$ majority of which were haptomonads $(65 \%)$ and a few metacyclics (5\%). Parasites from the well containing 1:1000 anti-LPG MABS showed mainly the longer, broader procyclics $(60 \%)$ and nectomonads (40\%). While those previously mixed with 1:100 anti-LPG MABS showed mainly the longer, broader procyclics $(90 \%)$ and very few nectomonads $(10 \%)$. At 36 hours in culture majority of the parasites $(80 \%)$ in the control well had already 
reached the metacyclic stage. This was statistically significant compared to all the other wells $(\mathrm{p}<0.05)$. Those previously mixed with undiluted mouse sera showed fewer nectomonads (5\%) majority of which were haptomonads (35\%) and metacyclics (60\%). In the well containing 1:1000 anti-LPG MABs majority of the parasites were procyclics (10\%), nectomonads (30\%) but mainly haptomonads (40\%) and fewer metacyclics (20\%). At this stage one could still see longer, broader procyclic forms (85\%) and nectomonads (10\%) and fewer haptomonads $(5 \%)$ in the well containing 1:1000MABS. This was statistically significant compared to the control well $(\mathrm{p}<0.5)$. The procyclics were still observable in this group even after 48 hours post-incubation.

Table 1

Percentage of the parasite forms observed at 12, 24 and 36 hours of culture

\begin{tabular}{|c|c|c|c|c|c|c|c|c|c|c|c|c|}
\hline \multirow[t]{2}{*}{ Incubation medium } & \multicolumn{3}{|c|}{$12 \mathrm{hrs}$} & \multicolumn{5}{|c|}{$24 \mathrm{hrs}$} & \multirow[b]{2}{*}{$\mathrm{P}$} & \multicolumn{3}{|c|}{$36 \mathrm{hrs}$} \\
\hline & $\mathrm{P}$ & $\mathrm{N}$ & $\mathrm{H}$ & M & $\mathrm{P}$ & $\mathrm{N}$ & $\mathrm{H}$ & M & & $\mathrm{N}$ & $\mathrm{H}$ & M \\
\hline Controls & 90 & 10 & 0 & 0 & 5 & 20 & 75 & 0 & 0 & 5 & 15 & 80 \\
\hline Undiluted mouse sera & 15 & 80 & 5 & 0 & 10 & 20 & 65 & 5 & 0 & 5 & 35 & 60 \\
\hline 1:100 anti-LPG MABS & 100 & 0 & 0 & 0 & 90 & 10 & 0 & 0 & 85 & 10 & 5 & 0 \\
\hline 1:1000 anti-LPG MABS & 90 & 10 & 0 & 0 & 60 & 40 & 0 & 0 & 10 & 30 & 40 & 20 \\
\hline
\end{tabular}

$\mathrm{P}=$ Procyclic promastigotes, $\mathrm{H}=$ Haptomonads, $\mathrm{N}=$ Nectomonads, $\mathrm{M}=$ Metacyclics

Table 2

Parasite forms at six days post-feeding in P-duboscqi fed on blood containing different antibodies

Sand flies $(\%)$ that supported the various promastigote forms

\begin{tabular}{|c|c|c|c|c|c|}
\hline $\begin{array}{l}\text { Incubation } \\
\text { medium }\end{array}$ & $\begin{array}{l}\text { No. of sand } \\
\text { and infected }\end{array}$ & $\begin{array}{l}\text { fed } \\
\text { Procyclis }\end{array}$ & Nectomonads & Haptomonads & Metacyclics \\
\hline Controls & 26 & $0(0)$ & $12(46)$ & $17(65)$ & $21(81)$ \\
\hline Undiluted mouse sera & 23 & $0(0)$ & $10(44)$ & $17(74)$ & 19)82) \\
\hline 1:100 anti-LPG MABS & 25 & $20(80)$ & $22(88)$ & $10(40)$ & $4(16)$ \\
\hline 1:1000 anti-LPG MABS & 20 & $5(25)$ & $18(90)$ & $16(80)$ & $13(65)$ \\
\hline
\end{tabular}

Separation of proteins on SDS-PAGE: Electrophoresis of gut homogenates and L. major parasites revealed a complexity of proteins consisting of 12 and 18 major bands respectively (Figure 1). When the sand fly gut homogenates were compared to purified L. major-derived LPG the two antigens showed common proteins of $105 \mathrm{kDa}$ and $106 \mathrm{kDa}$.

\section{Figure 1}

Separation of L. major parasites, sand fly guts Iysate and LPG following SDS -PAGE

Lanes 1 and 2: non-reduced and reduced forms of $P$. duboscqi gut Iysates, respectively; Lanes 3 and 4 non-reduced and reduced forms of purified $L$. major derived-LPG molecule, respectively. 
Parasite development in P. duboscqi sand flies which had previously fed anti-LPG MABS: Sand flies which fed on anti-LPG MABS had mainly procyclics and significantly very few metacyclics compared to control sand flies on day 6 post feeding $(\mathrm{p}<0.05)$ (Table 2). Very few sand flies, which had previously fed on 1:1000 anti-LPG MABS supported procyclic promastigotes but majority of the sand flies from this group, supported full parasite development up to the metacyclic stage. In sand flies, which had previously fed on undiluted mouse sera or amastigotes alone (controls), parasite development followed the normal developmental stages up to the metacyclic stage.

The results of sand fly dissections also indicated that sand flies, which had fed on anti-LPG MABS, showed low parasitemia levels, compared to their controls. Sand flies, which fed on blood meals containing amastigotes, mixed with 1: 100 showed the presence of parasites ranging from 1-10 parasites in 10-fields to 1-100 parasites in 10 fields. That is a range of grades of $2+$ to $3+$ according to the methods of Chulay and Brycesson (14) and modified by Tonui and colleagues (8). Sand flies, which had fed on amastigotes, mixed with al: 1000 anti-LPG MABS showed 1-10 parasites per field (4+). The control sand flies which fed on amastigotes alone to those which fed on amastigotes mixed with undiluted mouse sera showed parasites which ranged from 10 to 100 parasites per field (grades $5+$ and 6+).

\section{DISCUSSION}

Interactions between the blood feeding arthropods and their hosts may have profound implications for the transmission of vector-borne pathogens. Such host-parasite interactions are mediated by specialised molecules, which hold the key to our understanding of the intricacies of parasitism(17). A dense surface layer of glycolipids coats the parasitic protozoan Leishmania. Apart from showing protective functions these molecules act as developmentally regulated virulence factors involved in the recognition and attachment of host macrophages as well as binding the gut of the sand fly vector(5).

In this study, MABS raised against L. major -derived LPG were tested for their potential to reduce L. major development in vitro and within the sand fly. Results showed that blood mixed with 1:100 anti-LPG MABS showed significant numbers of procyclic promastigotes, three days in culture or in sand flies after six days post-feeding $(\mathrm{p}<0.05)$. These observations suggest that blood mixed with 1:100 MABS were capable of inhibiting L. major development at the procyclic stage both in vitro and within the sand fly. However, limited inhibition was seen when undiluted sera from $L$. major infected mice or 1:1000 anti-LPG MABS were used in vitro.
In our laboratory, the L. major parasite (strain $\mathrm{NLB}=144$ ) has been observed to be a fast growing parasite capable of reaching the metacyclic stage between 12-24 hours in culture (Tonui, personal observations). Transformed L. mexicana amastigotes have also been observed to have a life span of only three days in culture(18). Within the sand fly the procyclic stage is usually seen two three days postfeeding(2). The short life span in culture may be as a result of requirement for some factor or factors normally present in the intracellular milieu.

Low parasitemia levels were also detected in the guts of sand flies previously fed on MABS compared to their controls. Low numbers of metacyclic promastigote formation in sand flies has been shown to be responsible for reduced transmission of $L$. major to naive $\mathrm{BALB} / \mathrm{c}$ mice(8) and induction of resistance to the disease(19). The inhibition of $L$. major development by anti-LPG MABS may be explained in terms of the cyclical transformation of the LPG molecule during the process of metacyclogenesis within the sand fly(2). A high level of L. major inhibitions would have been expected with sera from $L$. major infected mice as a result of the many epitopes that can be recognised by these polyclonal sera, but instead a low level of inhibition was observed. The LPG molecule plays a major role during the process of metacyclogenesis by forming a protective coat against digestive enzymes and contains the ligand recognised in the attachment to the mid gut epithelial cells(6). Recent studies using mutant LPGI-deficient promastigotes concluded that the LPG is not essential for survival in the early blood fed mid gut, but along with other secreted phosphoglycan containing glycoconjugates, can protect promastigotes from the digestive enzymes in the gut; secondly, that LPG is required to mediate mid gut attachment and to maintain infection in the fly during excretion of the digested blood meal(20).

While the involvement of cell-mediated immune protective mechanisms has been demonstrated in leishmaniasis, the role of humoral mechanisms in protection is not clearly understood. However, in vitro effects of anti-leishmanial antibodies on promastigotes from super-infected mice or from nonimmune humans have been demonstrated(21). MABS directed against Leishmamia promastigotes have also been shown, using a Winn type assay system, to protect $\mathrm{BALB} / \mathrm{c}$ mice against challenge with promastigotes(22). Similar results were obtained with amastigote infection, and cross-species protection was demonstrated(23). Antibodies and effector cells of the immune system ingested with a blood meal, may affect vector functions in a number of ways. While it is unlikely that ingested leucocytes remain active for a long time, they may release cytokines and other, possibly toxic substances, especially if they are already activated by an infection in the vertebrate(24). It is not known, however, at what stage 
antibodies act on the parasite in the sand fly but since antibodies ingested in a blood meal persist intact in sand flies for several days, it may be that, during this time, they are able to affect amastigote transformation at days one to two post-infective blood meal. Persisting antibodies may then interfere with nectomonads, haptomonads and paramastigotes and thus reduce their transformation. Results from this study, suggests a possible role of humoral mechanisms in protection against leishmaniasis and which may be potentially useful in reducing parasite development in the sand fly.

How MABS raised against LPG caused inhibition both in culture and within the sand fly is however, unknown but certainly the presence of anti- LPG MABS in culture or in the mid gut might have caused interference to the process of metacyclogenesis. This may be supported by a report that a phosphorylated oligosaccharride repeat unit P04-6 (Gal \{beta 13\}) Gal \{1-4\} Man alphal- P3, specific for L. major was shown to be highly immunogenic and capable of inhibiting attachment of procyclic promastigotes to the mid gut of the sand fly vector(25). These results strongly support the view that LPG molecule is a promising candidate as a transmission blocking vaccine in leishmaniasis(8).

We have previously demonstrated in our laboratory that BALB/c mice immunised with a cocktail vaccine composed of a mixture of $L$. major flagella and $P$. duboscqi gut antigens significantly protected mice against a challenge infection with virulent $L$. major(26). In a related experiment, the inhibitory effect and sand fly mortality rates by antibodies raised against L.major flagella or sand fly gut antigen when the two were ingested separately was low compared to when the two were mixed together in a single blood meal(26). All these reports suggested that the sand fly gut homogenates are potential immunogens.

This preliminary study has shown that the sand fly has two proteins of $105 \mathrm{kDa}$ and $106 \mathrm{kDa}$ with similar molecular weights with those of the Leishmania parasite. It would be very interesting in future to isolate these fractions and explore their potential as vaccine candidates in leishmaniasis. If some of these molecules prove to be immunogenic molecular approaches would then be employed to identify the genes that express these proteins. If genes that are directly involved in LPG binding in the sand fly gut are isolated, this may open up a new way of combating leishmaniasis as blocking of these genes which are crucial for binding the Leishmania parasite in the sand fly mid gut may effectively disrupt development and hence transmission of the parasite.

\section{ACKNOWLEDGEMENTS}

To Mr. C. Were, Mr. P. Mwanyumba, Mr. S. Odongo from the Kenya Medical Research Institute (KEMRI); Dr. M. Gicheru and Mr. C. Kyama from the Institute for Primate Research (IPR), Nairobi, Kenya; Mr. D.
Kobowen and Mr. J. Laanoi of Marigat for their Technical assistance; Dr. R. Titus and Dr. D. Gillespie of Colorado State University for meaningful discussions on SDS-PAGE. This study received financial support in part from the International Atomic Energy Agency (IAEA), International Society for Infectious diseases (ISID), and KEMRI. This work is published with approval from the Director, KEMRI.

\section{REFERENCES}

1. Solbach, W. and Laskay, T. The host response to Leishmania infection. Adv. Immunol. 2000; 74:275-317.

2. Lawyer, P.G., Ngumbi, P.M., Anjili, C.O., et al. Development of Leishmania major and Sergentomyia schwetzi (Diptera: Psychodidae). J. Amer. Trop. Med. and Hyg. 1990; 43: 31-43.

3. McConville, M. J. and Ferguson, M.A. The structure, biosynthesis and function of glycosylated phosphatidylinositols in the parasitic protozoa and higher eukaryotes. Biochem. J. 1993; 294:305-324.

4. Lohman, K. L., Langer, P. J. and McMahon-Pratt, D. Molecular cloning and characterisation of the immunologically protective surface glycoprotein GP46/M2 of Leishmania amazonensis. Proc. Nat. Acad. Sci. 1990; 87:8393-8397.

5. Turco S. J., and Descoteaux, A. The lipophosphoglycan of Leishmania parasites. Ann. Rev. Microbiol. 1992; 46: 65-94.

6. Pimenta, P. F. P., Saraiva, M. B., Rowton, E., et al. Evidence that vectorial competence of Phlebotomine sand flies from different species of Leishmania is controlled by structural polymorphisms in the surface lipophosphoglycan. Proc. Nat. Acad. Sci. USA. 1994; 91:9155-9159.

7. Butcher, B.A., Turco, S.J., Hilty, B.A., et al. Deficiency in. 1,3-Galactosyl transferase of a Leishmania major LPG Mutant adversely influences the Leishmania-sand fly interaction. J. Biol. Chem.1996; 271:20573-20579.

8. Tonui, W. K. Leishmania transmission-blocking vaccines: a review. East. Afr. Med. J. 1999; 76:93-96.

9. Anjili, C. O., Mbati, P. A., Ngumbi, P. M., Lugalya, R. M. and Githure, J. I. Effects of feeding Phlebotomus duboscqi on different anti-Leishmania monoclonal antibodies on development of Leishmamia major. (2002 In Press.)

10. Tolson, D. L, Turco, S. J., Beecroft, R. P. and Pearson, T.W. The immunochemical structure and surface arrangement of Leishmania donovani lipophosphoglycan determined using monoclonal antibodies. Mol. Biochem. Parasitol. 1989; 35:109-118.

11. Kohler, G. and Milstein, C. Continous cultures of fused cells secreting antibody of predefined specificity. Nature. 1975; 256:497.

12. Kennett, R. H., Denis, K.A., Tung, A.S. and Klinman, N.R. Hybrid plasmacytoma production: fusion with adult spleen cells, monoclonal spleen fragments, neonatal spleen cells and human spleen cells. Curr. Topics in Microbiol. Immunol. 1978, 81:77.

13. Beach, R., Young, D. G. and Kiilu, G. New Phlebotomine sand fly colonies II. Laboratory colonisation of Phlebotomus duboscqi (Diptera: Psychodidae). J. Med. Entomol. 1986; 23:114-115.

14. Chulay, J.D. and Brycesson, A.D.M. Quantitation of amastigotes of Leishmania donovani in smears of splenic aspirates from patients with visceral leishmaniasis. Amer. J. Trop. Med. Hyg. 1983; 32:475-479. 
15. Laemli, U. K. Cleavage of structural proteins during the assembly of the head of bacteriophage T4. Nature. 1970; 227:680-685.

16. Towbin, H., Stachelin, T. and Gordon, J. Electrophoretic transfer of proteins from polyacrylamide amide gels to nitrocellulose sheets. Procedure and some applications. Proc. Nat. Acad. Sci. 1979; 76:4350-4354.

17. Ng, K., Handman, E. and Basic A., Biosynthesis of lipophosphoglycan from Leishmania major: solubilisation and characterisation of a (beta 1-3) galactosyltransferase. Biochem. J. 1996; 317:247-255.

18. Hunter, K. W. Jr, Cook, C. L., and Hensen, S. A. Temperature-induced in vitro transformation of Leishmania mexicana. I. Ultrastructural comparison of culturetransformed and intracellular amastigotes. Acta Tropica. 1982; 39:143-150.

19. Doherty, T.M. and Coffman, R.L. Leishmania major: Effect of infectious dose on T cell subset development in BALB/ c mice. Exp. Parasitol. 1996; 84:124-135.

20. Sacks, D.L., Modi, G., Rouston, E. et al. The role of phosphoglycans in Leishmania-sand fly interactions. Proc. Nat. Acad. Sci. 2000; 97:406-411.
21. Herman, R. Cytophilic and opsonic antibodies in visceral leishmaniasis in mice. Infect. Imm. 1980; 28:585-593.

22. Anderson, S., David, J.R. and McMahon-Pratt D. In vivo protection against Leishmania mexicana mediated by monoclonal antibodies. J. Immunol. 1983; 131:1616-1618.

23. Monjour, L., Vouldoukis, I., Debons-Guillemin, M. C., et al. Application of an in vivo culture system for rapid demonstration of anti-Leishmania activity of monoclonal antibodies. Trans. Roy. Soc. Trop. Med. Hyg. 1987; 81:210-211.

24. Ramasamy, M. S., and Ramasamy, R. Effect of antimosquito antibodies on the infectivity of the rodent malaria parasite Plasmodium berghei to Anopheles farauti. Med. Vet. Entomol. 1990; 4:161-166.

25. Kelleher, M., Curtis, J.M., Sacks, D.L., Handman, E. and Basic, A. Epitope mapping of monoclonal antibodies directed against lipophosphoglycan of Leishmania major promastigotes. Mol. Biochem. Parasitol. 1994; 66:187-200.

26. Mbati, P.A., Anjili, C.O., Lugalia, R., et al. Experimental immunisation against cutaneous leishmaniasis using Leishmania major subcellular fractions alone or in combination with Phlebotomus duboscqi gut antigens. East Afr. Med. J. 1995; 72:519-522. 